\title{
Possible re-infection of SARS-CoV-2 complicated by dengue virus co-infection: report of a rare case from Bangladesh
}

\author{
Wahiduzzaman $\mathbf{M}^{\mathrm{a}}$, Rahim MA ${ }^{\mathrm{b}}$
}

\begin{abstract}
Re-infection by severe acute respiratory syndrome coronavirus 2 (SARS-CoV-2) and co-infection by dengue virus and SARS-CoV-2 are possible. We report a case of dengue haemorrhagic fever, occurring in a young Bangladeshi man, who concurrently tested positive for SARS-CoV-2 infection by reverse transcriptase polymerase chain reaction (RT-PCR). Four months previously, he suffered a mild form of corona virus disease 2019 (COVID-19). This case is reported to make the physicians aware that, co-infections are possible in this COVID-19 pandemic, specially in dengue endemic regions and countries like Bangladesh.
\end{abstract}

Key words: COVID-19, dengue haemorrhagic fever, re-infection, RT-PCR positivity.

(BIRDEM Med J 2021; 11(1): 105-106)

\section{INTRODUCTION}

Starting from Wuhan, China in late 2019, severe acute respiratory syndrome coronavirus 2 (SARS-CoV-2) has rapidly spread all over the world and claimed huge morbidity and mortality. These are not only due to corona virus disease 2019 (COVID-19) itself but also from other diseases including concurrent infections. In tropics and sub-tropical countries, dengue outbreaks are common during the monsoon. Initial clinical and laboratory features are indistinguishable for dengue and COVID-19. ${ }^{1}$ Here, we present a case of dengue haemorrhagic fever, occurring in a young Bangladeshi male patient, who also tested positive for SARS-CoV-2 during the same febrile illness by reverse-transcriptase polymerase chain reaction (RT-PCR), four months after his first clinical event of COVID-19.

\section{CASE REPORT}

A 34-year-old Bangladeshi male patient, with no recent travel history, presented with a 2-day history of high grade, continued fever, headache, body ache, low back pain and restlessness. He took paracetamol and fexofenadine. Investigation reports revealed a positive natural structural protein 1 (NS1) for dengue virus and RT-PCR for SARS-CoV-2 from nasopharyngeal swabs. His initial platelet count was $1,70,000 / \mathrm{cmm}$ of blood. He was started with favipiravir, advised to stay home and monitor oxygen saturation. High resolution computed tomography (HRCT) scan of chest appeared normal. On day 4 of symptom onset, he developed wide spread maculo-papular rash. A repeat blood test revealed low platelet count $(42,000 / \mathrm{cmm})$ and he was hospitalized, where subsequently his platelet count further dropped to $28,000 / \mathrm{cmm}$. He remained clinically stable throughout the hospital course; there was no bleeding manifestation, shock or hypoxia. His discharge medications included subcutaneous low molecular weight heparin (started after platelet count had reached over $1,00,000 / \mathrm{cmm})$.

\section{Author information}

a. Miah Wahiduzzaman, Deputy Chief Medical Officer, Outpatient Department, BIRDEM General Hospital, Dhaka, Bangladesh.

b. Muhammad Abdur Rahim, Associate Professor, Department of Nephrology, BIRDEM General Hospital, Dhaka, Bangladesh.

Address of correspondence: Miah Wahiduzzaman, Deputy Chief Medical Officer, Outpatient Department, BIRDEM General Hospital, Dhaka, Bangladesh. Email: miahwahiduzzaman@yahoo.com, muradrahim23@yahoo.com

Received: November 1, 2020

Revision received: November 12, 2020

Accepted: December 20, 2020 
On June 23, 2020, he first reported with a 3-day history of fever, cough, sore throat and anosmia. That time, he tested positive by RT-PCR for SARS-CoV-2 and was treated at home as mild COVID-19 case, maintaining isolation protocols and took paracetamol, fexofenadine, azithromycin and single dose of ivermectin. He became symptom free in 5 days and tested negative for SARSCoV-2 after 21 days. He remained in good health throughout the period until October 15, when he again developed febrile illness and subsequently received a diagnosis of dengue haemorrhagic fever and COVID19.

On follow-up visit, one week after discharge, he was clinically stable and laboratory evaluation revealed positive IgG against dengue virus (15 days after fever onset, IgM and IgG were negative on day 5 after fever onset), improved alanine aminotransferase (151 from $444 \mathrm{U} / \mathrm{L})$ and a normal platelet count and D-dimer level.

\section{DISCUSSION}

Antibodies against SARS-CoV-2 are not long lasting and re-infection by SARS-CoV-2 is possible. ${ }^{2}$ Cases of co-infection by dengue virus and SARS-CoV-2 are reported from Reunion Island ${ }^{1}$ and Brazil. ${ }^{3}$ Care should be taken to make a diagnosis of co-infection by dengue virus and SARS-CoV-2 depending up on serology, as false positive dengue serology in COVID-19 cases are reported. 4

It is now evident that co-infections are possible by dengue virus and SARS-CoV-2. Some authors warn about the worse combination, while others predict some protective role, at least short-lived ones. Important is to treat the patient by supportive measures, with meticulous monitoring for possible organ involvement/failure, protection of caregivers and hospital staffs from SARSCoV-2 infection. ${ }^{5,6}$

Repeat infections by SARS-CoV-2 are reported. In our clinical practice, clinical manifestation and second time RT-PCR positivity for SARS-CoV-2 after recovery from the first event are documented but we are unable to go for genome sequencing as routine practice. This limits the proof for re-infection in resource constrain settings, as in the present case. It is also suggested that, in absence of clinical features, RT-PCR positivity from nasopharyngeal swabs does not necessarily mean persistent infection, re-infection or infectivity. ${ }^{7}$

As COVID-19 cases are increasing with evidences of second waves in some countries and regions, it will not be surprising to face increasing numbers of re-infection by SARS-CoV-2 and co-infection with dengue virus and SARS-CoV-2. A high index of clinical suspicion is warranted to have a better patient outcome and safety for health care workers.

Authors' contribution: MW diagnosed the case and planned publication. MAR did literature search and drafted the manuscript. Both authors read and approved the final manuscript for publication.

Conflicts of interest: Nothing to declare.

Consent: Informed written consent was obtained from the patient for publication of case report and accompanying images.

\section{REFERENCES}

1. Verduyn M, Allou N, Gazaille V, Andre M, Desroche T, Jaffar M-C, et al. Co-infection of dengue and COVID-19: A case report. PLoS Negl Trop Dis 2020; 14(8): e0008476.

2. Tillett RL, Sevinsky JR, Hartley PD, Kerwin H, Crawford N, Gorzalski A, et al. Genomic evidence for reinfection with SARS-CoV-2: a case study. Lancet Infect Dis 2020 Published Online October 12, 2020 https://doi.org/10.1016/S14733099(20)30764-7

3. Bicudo N, Bicudoa E, Costab JD, Castroa JALP, Barra GB. Co-infection of SARS-CoV-2 and dengue virus: a clinical challenge. Braz J Infect Dis 2020; 24(5): 452-4.

4. Yan G, Lee CK, Lam LTM, Yan B, Chua YX, Lim AYN, et al. Covert COVID-19 and false-positive dengue serology in Singapore. Lancet Infect Dis 2020 May; 20(5): 536.

5. Ridwan R. COVID-19 and dengue: a deadly duo. Tropical Doctor 2020; 50(3): 270-2.

6. Joob B, Wiwanitkit V. COVID-19 in medical personnel: observation from Thailand. J Hosp Infect 2020; 104: 453.

7. Rahim MA, Mostafi M. Repurposive Use of Drugs in COVID19: A Wake-up Call. J Bangladesh Coll Phys Surg 2020; 38 (Suppl. Issue): 3-4. 